\title{
Investigação do Uso de Parâmetros Biofísicos na Influência do Microclima Dentro de Áreas Urbanas
}

\section{Biophysical Parameters Investigation in the Influence of Microclimates in Urban Areas}

\author{
Denise Pereira Canedo Meira Vieira*a; Victor Hugo de Morais Danelichena; Mariane Batista de Lima Moraes Brandão \\ Campos $^{\mathrm{a}}$
}

aUniversidade de Cuiabá, Programa de Pós-Graduação Stricto Sensu em Ciências Ambientais. MT, Brasil.

*E-mail: canedodenise@hotmail.com

\begin{abstract}
Resumo
Diante da necessidade de obtenção de informações relacionadas ao microclima e influência da vegetação dentro de um perímetro urbano na qualidade de vida dos seus habitantes se define como parâmetros biofísicos a serem estudados nesta pesquisa o Normalized Difference Vegetation Index (NDVI) e Leaf Area Index (LAI). Considerando que o Sensoriamento Remoto é uma tecnologia de baixo custo e fácil aquisição podendo ser obtidas gratuitamente, via banco de dados disponíveis na Internet, verifica-se que o sensoriamento pode ser utilizado como fonte confiável de levantamento desses parâmetros. Este estudo tem como objetivo analisar a produção científica sobre o uso do sensoriamento remoto como tecnologia alternativa para obtenção de informações de parâmetros biofísicos, como o NDVI e LAI, possibilitando a preservação e o planejamento da vegetação nos espaços urbanos. O artigo se trata de uma revisão narrativa realizada através de consultas aos bancos de dados Scientific Electronic Library Online (SciELO), Literatura Latino-Americana e, principalmente, do banco de dados Scopus (Elsevier) da CAPES. Como critérios de inclusão foram aplicados: artigos com disponibilidade completa de 2010 até 2020 e com relação direta com o estudo. É possível concluir que como o ambiente sofre alterações constantes pela ação antrópica e a interpretação de imagens de satélite é uma fonte direta de se determinar a dinâmica dos processos envolvidos em tais alterações, a fotointerpretação e o processamento digital de imagens assumem papel de grande importância. Tais ferramentas permitem fornecer subsídios para a compreensão dos fenômenos ambientais, além da possibilidade de planejamento estratégico no planejamento urbano. As revisões bibliográficas realizadas indicam a fotointerpretação e o processamento digital de imagens como ferramentas ainda pouco utilizadas para estimar os parâmetros biofísicos no contexto urbano.
\end{abstract}

Palavras-chave: Sensoriamento Remoto. Espaços Urbanos. NDVI e LAI.

\begin{abstract}
Given the need to obtain information related to the vegetation microclimate and influence within an urban perimeter on the quality of life of its inhabitants, it was defined as biophysical parameters to be studied in this research the Normalized Difference Vegetation Index (NDVI) and Leaf Area Index (LAI). Considering that Remote Sensing is a low cost and easy acquisition technology and can be obtained free of charge via the database available on the Internet, it will be verified that sensing can be used as a reliable source of survey of these parameters. This study aims to analyze the scientific production on the use of remote sensing as an alternative technology to obtain information from biophysical parameters, such as NDVI and LAI, enabling the vegetation preservation and planning in urban spaces. The article is a narrative review carried out through consultations with the Databases Scientific Electronic Library Online (SciELO), Latin American Literature and mainly the Scopus database (Elsevier) of CAPES. Inclusion criteria were applied: articles with complete availability from 2010 to 2020 and with direct relation to the study. It is possible to conclude that the environment undergoes constant changes by anthropic action and the satellite images interpretation is a direct source for determining the processes dynamics involved in such changes, photointerpretation and digital image processing play a major role. Such tools allow to provide subsidies for the understanding of environmental phenomena, in addition to the possibility of strategic planning in urban planning. The bibliographic reviews performed indicate photointerpretation and digital image processing as tools still little used to estimate biophysical parameters in the urban context.
\end{abstract}

Keywords: Remote Sensing. Urban Spaces. NDVI and LAI

\section{Introdução}

O acelerado crescimento urbano, a consequente alteração da paisagem e das características ambientais, principalmente, nas grandes cidades, têm gerado uma série de problemas que se relacionam diretamente com a qualidade ambiental e a qualidade de vida de suas populações (NASCIMENTO et al., 2013). Além disso, o aumento do número de pessoas nas áreas urbanas está ameaçando os ecossistemas, pois a urbanização é acompanhada por uma selagem maciça do solo, a densificação das áreas construídas e a perda e degradação relacionadas dos espaços verdes urbanos (KABISCH et al., 2017).

Os departamentos de planejamento nas cidades dependem de informações atualizadas sobre o uso da terra para planejar e tomar decisões e políticas mais qualificadas sobre onde desenvolver espaços residenciais, infraestrutura e, também, onde manter e desenvolver novos espaços verdes urbanos para melhorar a saúde e o bem-estar dos moradores. À medida que as áreas urbanas estão aumentando em número e densidade com alterações em áreas construídas e impermeáveis, a capacidade 
de monitorar essas mudanças é de extrema importância e se tornará mais crítica no futuro (KABISCH et al., 2018).

Nesse sentido, o sensoriamento remoto em função de seu caráter sinóptico e rapidez na aquisição de dados, destaca-se como uma técnica capaz de monitorar as culturas ao longo de seu ciclo de vida (OLDONI et al., 2019). Esses dados podem ser usados para esses fins ou mesmo em uma aplicação econômica, por exemplo, para a indústria da construção, logística ou seguro. Como a abordagem é de natureza genérica, permite a integração quase em tempo real de outros dados para otimizar e prever relacionamentos e processos complexos em sistemas urbanos heterogêneos.

No monitoramento das culturas, é possível computar índices de vegetação como o NDVI (Normalized Difference Vegetation Index) e LAI (Leaf Area Index), que trazem informações importantes sobre a vegetação e possibilita a tomada de decisões pelo poder público responsável pela gestão urbana. Segundo Soares et al. (2020) se torna necessário o desenvolvimento de trabalhos, que utilizem técnicas e métodos que permitam analisar os processos de degradação da paisagem nativa de uma região, de modo que este possa ser utilizado no estabelecimento de políticas e estratégias, cujo objetivo seja o desenvolvimento sustentável. Assim, o NDVI derivado de diferentes informações do sensor pode ser usado para detectar processos de densificação em pequena escala ou alterações na cobertura de espaços verdes, mesmo em áreas urbanas. Ainda assim, a gama de valores é diferente quando são consideradas estruturas urbanas altamente diversificadas com alta complexidade de vegetação verde com ruas não verdes, prédios ou solo descoberto (GASCON et al., 2016).

Diante disso, o objetivo deste trabalho é investigar a utilização do sensoriamento remoto como ferramenta promissora nos estudos envolvendo parâmetros biofísicos dentro de um perímetro urbano.

\section{Desenvolvimento}

\subsection{Metodologia}

O presente artigo se trata de uma revisão bibliográfica realizada por meio de consultas aos bancos de dados Scientific Electronic Library Online (SciELO) e outros artigos disponíveis em revistas online, no google acadêmico e, ainda, no portal da CAPES. Neste último se utiliza o endereço (http:// www-periodicos-capes-gov-br.ez111.periodicos.capes.gov. br/), sendo feita a busca na base Scopus (Elsevier). Em seguida, se inseriu o TÍTULO-ABS-CHAVE (NDVI AND LAI), o que deu um resultado de 1252 documentos. Refinou-se seguindo os seguintes parâmetros: anos de 2015 até 2020, área da matéria de Ciências Ambientais, tipo de documento artigo, gerando um resultado de 94 artigos, cujos resumos foram todos lidos. Selecionaram-se então 42 artigos publicados entre 2015 e 2020, da área de ciências ambientais, que se utilizavam de imagens de satélite para medir ou calcular os índices NDVI e LAI e estes foram lidos integralmente.

\subsection{O Parque Urbano}

Sabe-se que um Parque, como Unidade de Conservação, tem como objetivo básico a preservação de ecossistemas naturais de grande relevância ecológica e beleza cênica, possibilitando a realização de pesquisas científicas e o desenvolvimento de atividades de educação e interpretação ambiental, de recreação em contato com a natureza e de turismo ecológico (FUJACO et al., 2010).

Segundo Nascimento (2013), a manutenção do verde vem sendo justificada pelo seu potencial em realçar aspectos associados à qualidade ambiental, enquanto provedora de benefícios ao homem, interferindo positivamente na qualidade de vida pela manutenção das funções ambientais, sociais e estéticas, amenizando as propriedades negativas do processo de urbanização.

A minimização das ilhas de calor e melhoria do conforto em função das áreas verdes é também um dos grandes benefícios dos parques urbanos, sendo a qualidade de vida do entorno desses parques diretamente proporcional a área de vegetação desses. Logo, o acompanhamento constante da vegetação dos parques se faz necessário e o monitoramento ambiental, em escala regional, pode ser realizado, a partir de técnicas de sensoriamento remoto, as quais permitem analisar a relação entre padrões espaciais da vegetação e as mudanças no balanço de radiação e dos fluxos de energia da superfície (FAUSTO et al., 2014).

\subsection{Sensoriamento remoto}

De acordo com Pirovani (2012), as tecnologias espaciais são importantes ferramentas, com destaque para as imagens de sensoriamento remoto, que possibilitam a observação em tempo real de remanescentes florestais, análise espaçotemporal das transformações da paisagem, avaliação dos padrões de uso e cobertura da terra, quantificação da estrutura e padrões de distribuição da paisagem. $\mathrm{Na}$ execução dos tipos de análises citadas é indispensável a utilização do Sistema de Informação Geográfica (SIG), que proporciona a extração de dados das imagens de sensoriamento remoto e a organização destes em Banco de Dados Geográficos (BDG) (COCHEV et al., 2019).

Do site do INPE (http://www.dgi.inpe.br/documentacao/ satelites/landsat) se conseguem as informações sobre a série Landsat (Land Remote Sensing Satellite), que teve início na segunda metade da década 1960, a partir de um projeto desenvolvido pela Administração Nacional de Aeronáutica e Espaço (NASA), sendo dedicado exclusivamente à observação dos recursos naturais terrestres. Esse programa foi inicialmente denominado ERTS (Earth Resources Technology Satellite) e, em 1975, passou a se denominar LANDSAT. De lá até hoje foram lançados 8 satélites da série, todos com imagens multiespectrais. O LANDSAT-1 foi o primeiro satélite e, também, o primeiro desenvolvido para atuar, diretamente, em pesquisas de recursos naturais, foi lançado 
em 1972 e denominado ERTS-1 ou LANDSAT-1. Foi o primeiro satélite de sensoriamento remoto no Mundo e levou dois instrumentos a bordo, as câmeras RBV e MSS. Operou por um período de cinco anos adquirindo mais de 300.000 imagens, com repetidas coberturas da superfície da Terra. De lá até hoje foram lançados 8 satélites da série, todos com imagens multiespectrais. No catálogo do INPE se encontram imagens do sensor Multispectral Scanner (MSS) $80 \mathrm{~m}$ resolução espacial, para os satélites LANDSAT 1,2 e 3 (19721992), do sensor Thematic Mapper (TM) de 30m resolução espacial, no satélites LANDSAT-5 (1984-2011) e o sensor LANDSAT Enhanced Thematic Mapper Plus (ETM +) de 15 a $30 \mathrm{~m}$ de resolução, no satélite LANDSAT-7 (desde 1999) e do sensor OLI (Operational Terra Imager) e TIRS (Thermal Infrared Sensor) 15 a 30 metros de dados multiespectrais do LANDSAT-8 (Desde 2013). As imagens do LANDSAT-8 são ortoretificadas e todos os produtos do catálogo estão em formato GeoTIFF.

Logo, se têm informações suficientes, com qualidade e gratuitas para serem utilizadas no desenvolvimento de uma investigação de parâmetros biofísicos em uma determinada região.

\section{4 Índices de Vegetação - NDVI e LAI}

Entre os índices mais citados na bibliografia brasileira está o Índice de Vegetação da Diferença Normalizada (NDVI - Normalized Difference Vegetation Index), utilizado para avaliação das mudanças do vigor vegetativo das plantas objetivando a correlação com outros parâmetros como o índice de área foliar, a biomassa ou a produtividade da vegetação (GALVANIN et al., 2014). O NDVI permite um diagnóstico detalhado da fitomassa fotossinteticamente ativa, corrigindo os efeitos de sombras causados pela elevação da topografia nas imagens de satélite, sendo por isso um índice muito usado e aceito para analisar a cobertura vegetal utilizando o sensoriamento remoto. Ainda, o índice de vegetação com diferença normalizada (NDVI) é o VI mais utilizado para a estimativa de IAF, mas é suscetível à refletância do solo, quando os valores de IAA são relativamente baixos e sua sensibilidade diminui rapidamente com o aumento da IAE (GU et al., 2013).

Segundo Berger (2019), o Índice de área Foliar, estrutura do dossel vegetativo, são importantes na produtividade da vegetação e no consumo de água da mesma, estando, portanto, correlacionada às trocas de umidade entre o ar e a vegetação, impactando diretamente nas variáveis de conforto ambientais. O Índice de área foliar (IAF ou LAI, inglês), pode ser obtido de várias maneiras, utilizando os métodos diretos e os indiretos. Entretanto, os métodos diretos consomem muito tempo em sua determinação, tornando-os praticamente inviáveis para seu uso em grande escala. Por outro lado, os métodos indiretos são de mais fácil utilização, sendo que normalmente os métodos óticos são os preferidos, por sua relativa rapidez e precisão. Muitos estudos extraíram seus valores de IAF de modelos fenológicos (por exemplo, MURRAY-TORTAROLO et al., 2013 ; CAO et al., 2015 ) ou dados baseados em satélite (por exemplo, SLEVIN et al., 2015), porque essas fontes são facilmente acessíveis e podem produzir resultados em etapas de tempo utilizáveis no espaço desejado.

\subsection{Relação entre os Índices de Vegetação e Sensoriamento Remoto}

A relação entre o sensoriamento remoto e os parâmetros biofísicos pode ser feita por meio das bandas simples e, também, pela aplicação de índices de vegetação. Os índices de vegetação são, frequentemente, utilizados para estimar parâmetros de vegetação, e a base física desses índices é atribuída à alta absorção da radiação pela clorofila e ao seu espalhamento pelas folhas, nas regiões espectrais do vermelho e do infravermelho próximo, respectivamente (GATES et al., 1965).

Para fontes de dados se pode utilizar uma série de imagens coletadas em um intervalo de tempo definido. Estas devem ser trabalhadas, pois uma das características inerentes às séries temporais é a presença de ruídos causados, por exemplo, no caso do sensor MODIS, por interferências da atmosfera (cobertura de nuvens) e/ou por ângulos de incidência do sensor relativamente elevados.

Diversos métodos de suavização de séries temporais têm sido propostos, tais como a média móvel, a mediana, a análise harmônica, a transformada de Fourier, a transformada de Wavelet ou o filtro Savitzky-Golay (CHEN et al., 2004; CARVALHO JR. et al., 2006; BRADLEY et al., 2007; LU et al., 2007; COUTO JR. et al.,2012). Cada filtro apresenta vantagens e desvantagens em relação às outras. Portanto, a escolha do filtro mais adequado para suavizar as séries temporais de interesse se constitui em uma das etapas de pré-processamento que deve ser considerada em estudos que envolvem essas séries.

Outro aspecto importante é o fato de que as séries temporais, geralmente, apresentam grande quantidade de dados. Por isso, é recomendável o uso de técnicas que reduzem a dimensionalidade dos dados, sem perdas significativas de informações, como a MNF. No Brasil, essa tem sido aplicada na análise de séries temporais de sensores multiespectrais e com resolução espacial moderada (CARVALHO JR. et al., 2012; COUTO JR. et al., 2013). Além disso, o método de minimum noise fraction (MNF), proposto por Green et al. (1988), leva em consideração os padrões espaciais repetitivos na série temporal para filtrar os dados. Dessa forma, a aplicação desta técnica, em séries temporais, elimina a sobreposição das informações dos pixels em imagens de diferentes datas.

O grande desafio, no uso de séries temporais, para produzir mapas de uso e cobertura vegetal consiste em lidar com as mudanças que ocorrem em tempos distintos, ao longo da série temporal, as quais estão relacionadas, por exemplo, com desmatamentos, queimadas, mudanças de usos e de práticas de manejo. Tal dinâmica implica em feições nas séries que 
não são inerentes ao comportamento dos alvos naturais, mas às intervenções antrópicas associadas às características dos alvos. Consequentemente, o uso das séries temporais tem sido enfatizado na identificação pontual de mudanças, ao longo dessas séries, que estariam associadas com as interferências humanas (ANJOS et al., 2013).

\section{Conclusão}

Entre os artigos pesquisados neste trabalho há poucos que utilizam essa tecnologia para levantar e estudar o LAI e NDVI, mesmo sendo viável sua utilização. De forma se vê a possibilidade de que o sensoriamento remoto seja uma ferramenta utilizada na coleta e medição dos parâmetros biofísicos da vegetação, pois permite a medição dessas interações eletromagnéticas com alvos à superfície através de bandas espectrais.

A utilização dos índices LAI e NDVI como parâmetros de acompanhamento e estudo das áreas verdes urbanas também foi bastante justificada pelos artigos analisados nesta pesquisa bibliográfica, pois a presença de áreas verdes se justifica pelos aspectos associados à saúde humana e aos fatores, que influenciam nas condições ambientais, como diminuição da temperatura e o aumento da umidade.

E ainda, de acordo com a pesquisa bibliográfica feita, as imagens do Landsat 8 podem ser escolhidas como fonte, bastante viável, de bandas espectrais para análise dos parâmetros biofísicos, pois são gratuitas, facilmente transferíveis e podem ser processadas facilmente. Entretanto, um pré-processamento das imagens se faz necessário, já que a cobertura vegetal de uma área sofre influência de fatores como o clima, a topografia, os solos e as suas propriedades, os valores de índice de vegetação, que podem sofrer significativas alterações nos períodos e estação do ano.

\section{Referências}

ANJOS, V. S. et al. Caracterização espectro-temporal de pastagens do triângulo mineiro utilizando dados MODIS EVI2 (2000-2010). Soc. Nat., v.25, n.1, p.205-215, 2013. doi: 10.1590/ S1982-45132013000100016

BERGER, R. et al. Índices de vegetação para a estimativa do Índice de Área Foliar em plantios clonais de Eucalyptus saligna Smith. Ciênc. Florestal, v.29, n.2, p.885-899, 2019. doi: $10.5902 / 1980509816942$

CAO, X. et al. Improving leaf area index simulation of ibis model and its effect on water carbon and energy: case study in changbai mountain broadleaved forest of china. Ecol. Modelling, v.303, p.97-104, 2015. doi: 10.1016/j.ecolmodel.2015.02.012

CARVALHO JR., O. A et al. Combining noise-adjusted principal components transform and median filter techniques for denoising Modis temporal signatures. Rev. Bras. Geofisica, v.30, n.2, p.147157, 2012. doi: 10.1590/S1982-21702014000200030

CESSA, R.M.A. Conforto térmico em áreas verdes na cidade de sorriso-MT. Rev. Soc. Bras. Arboriz. Urbana, v.2, n.1, p.17-30, 2017. doi: 10.5380/revsbau.v12i1.63487

CHEN, R. et al. VPR- Mediated Incorporation of UNG2 into HIV-1 Particles Is Required to Modulate the Virus Mutation Rate and for Replication in Macrophages. J. Biol. Chem., v.279, n.27, p.28419-28425, 2004. doi: 10.1074/jbc.M403875200

COUTO JÚNIOR, A.F.; CARVALHO JÚNIOR, O.A.; MARTINS, E.S. Séries temporais modis aplicadas em sucessão de culturas de soja (Glycine max (L.) Merrill) e milho (Zeamays L.) em sistema de plantio direto. Rev. Bras. Cartogr., v.64, n.3, p.405-418, 2012.

COCHEV, J.S. et al. Dinâmica espaço-temporal da paisagem e estrutura populacional de Euterpe precatória Mart. Em fragmento florestal no município mato-grossense de Alta Floresta, Brasil. Ciênc. Florestal, v.29, n.3, p.1398-1414, 2019. doi: $10.5902 / 1980509831737$

FAUSTO, M. A. et al. Net radiation estimated by remote sensing in Cerrado áreas in the Upper Paraguay River Basin. J. Appl. Remote Sensing, v.8, n.1, 2014. doi: 10.1117/1.JRS.8.083541

FUJACO, M.A.G.; LEITE, M.G.P.; MESSIAS, M.C.T. B. Análise multitemporal das mudanças no uso e ocupação do Parque Estadual do Itacolomi (MG) através de técnicas de geoprocessamento. REM, v.63, n.4, p.695-701, 2010. doi: $10.1590 / \mathrm{S} 0370-44672010000400016$

GATES, D.M. et al. Spectral properties of plants. Appl. Optics, v.4, n.1, p.11, 1965. doi: 10.1364/AO.4.000011

GASCON, M. et al. Índice de vegetação com diferença normalizada (NDVI) como marcador de verdura circundante em estudos epidemiológicos: o caso da cidade de Barcelona. Urbano para. Verde Urbano, v.19, p.88-94, 2016. doi: 10.1016/j. ufug.2016.07.001

GALVANIN, E.A.S. et al. Avaliação dos índices de vegetação NDVI, SR e TVI na discriminação de fitofisionomias dos ambientes do Pantanal de Cáceres/MT. Ciênc. Florestal, v.24, n.3, p.707-715, 2014. doi: 10.1590/1980-509820142403018

GREEN, A.A.M. et al. Uma transformação para solicitar dados multiespectrais em termos de qualidade de imagem com implicações para remoção de ruído. IEEE Transactions on Geoscie. Remote Sensing, v.26, n.1, p.65-74, 1988. doi: 10.1109/36.3001.

GU, Y. et al. Ajuste de saturação do NDVI: uma nova abordagem para melhorar as estimativas de desempenho das terras cultiváveis na bacia do Grande Platte, EUA. Ecol. Indic., v.30, p.1-6, 2013. doi: 10.1016/j.ecolind.2013.01.041

KABISCH N. et al. Soluções baseadas na natureza para adaptação às mudanças climáticas em áreas urbanas, teoria e prática de transições de sustentabilidade urbana. Springer Int. Publi., 2017.

KABISCH, N. et al. As cidades importam: espaços de trabalho nas avaliações de serviços ecossistêmicos com ferramentas de apoio à decisão no contexto dos sistemas urbanos. Bioscience, v.68, n.3, p.164-166, 2018. doi: 10.1093/biosci/bix153

NASCIMENTO, S.T.M.F.; RIBEIRO, E.S.; SOUSA, R.A.T.M.E. Valoração econômica de uma unidade de conservação urbana, Cuiabá, Mato Grosso. Interações (Campo Grande), v.14, n.1, p.79-88, 2013. doi:10.1590/S1518-70122013000100008

MURRAY-TORTAROLO, G. et al. Avaliação de modelos de superfície terrestre na reprodução de IAF derivada de satélite no hemisfério norte de alta latitude. Parte I: DGVMs não acopladas. Remote Sens., v.5, p.4819-4838, 2013. doi: 10.3390/ rs5104819

SOARES, J. et al. Estudo da dinâmica espaço-temporal do NDVI no Município de Sorriso-MT. Rev. Bras. Geog. Fís., v.13, n.2, p.834-841, 2020. doi: 10.26848/rbgf.v13.2.p834-841

SLEVIN, D.; TETT, S.F.B.; WILLIAMS, M. Multi-site evaluation of the JULES land surface model using global and 
local data. Geoscie. Model Develop., v.8, n.2, p.295-316, 2015. doi: 10.5194/gmd-8-295-2015

OLDONI, L.V. et al. Annual cropland mapping using data mining and OLI Landsat-8. Rev. Bras. Eng. Agríc. Amb., v.23, n.12, p.952958, 2019. doi: 10.1590/1807-1929/agriambi.v23n12p952-958
PIROVANI, D. Uso de geotecnologias para estudo da fragmentação florestal com base em princípios de ecologia da paisagem. In: SANTOS, R.A. et al. Geotecnologias aplicadas aos recursos florestais. Alegre: Caufes, 2012. p.24-41. doi: 10.1590/ S0100-67622014000200007 